\title{
Dots - An Inclusive Natural User Interfaces (NUI) for Spatial Computing
}

\author{
Weilun Gong \\ Imperial College London and Royal College of Art, United \\ Kingdom, weilun.gong@network.rca.ac.uk \\ Xiaohui Wang \\ Imperial College London and Royal College of Art, United \\ Kingdom, xiaohui.wang@network.rca.ac.uk
}

\begin{abstract}
The rise of ubiquitous computing and pervasive technology like Mixed Reality (MR) and the Internet of Things (IoT) causes the user interface starts to shift from the touch screens to the surrounding environment. However, the inclusive design has not adequately introduced the accessibility of these emerging technologies to people with physical disability. In this context, a new approach for these people seems necessary in relation to interface design for the sake of accessibility. Unlike traditional inclusive design where an object or device need to embrace different types of users, here we question whether users can adapt to one flexible and customizable system. In this paper, we present an "inclusive" Natural User Interface (NUI), which enables people with physical disabilities to interact with the spatial computing environment.
\end{abstract}

\section{CCS CONCEPTS}

- Human-centered computing $\rightarrow$ Human computer interaction (HCI); Interaction paradigms; Mixed / augmented reality; Accessibility; Accessibility technologies.

\section{KEYWORDS}

Inclusive design, wearable devices, natural user interface, spatial computing, assistive technology,

\section{ACM Reference Format:}

Weilun Gong, Lan Xiao, Xiaohui Wang, and Chang Hee Lee. 2020. Dots - An Inclusive Natural User Interfaces (NUI) for Spatial Computing. In 22nd International Conference on Human-Computer Interaction with Mobile Devices and Services (MobileHCI '20 Extended Abstracts), October 05-08, 2020, Oldenburg, Germany. ACM, New York, NY, USA, 4 pages. https://doi.org/10. $1145 / 3406324.3410715$

\section{INTRODUCTION}

Assistive technology and inclusive design for disabled people are well-studied areas, but, with the emerging technologies, they may face new challenges. Over the past few years, the development

Permission to make digital or hard copies of part or all of this work for personal or classroom use is granted without fee provided that copies are not made or distributed for profit or commercial advantage and that copies bear this notice and the full citation on the first page. Copyrights for third-party components of this work must be honored For all other uses, contact the owner/author(s).

MobileHCI '20 Extended Abstracts, October 05-08, 2020, Oldenburg, Germany

(c) 2020 Copyright held by the owner/author(s)

ACM ISBN 978-1-4503-8052-2/20/10.

https://doi.org/10.1145/3406324.3410715

\author{
Lan Xiao \\ Imperial College London and Royal College of Art, United \\ Kingdom, lan.xiao@network.rca.ac.uk \\ Chang Hee Lee \\ Royal College of Art, United Kingdom, \\ changhee.lee@network.rca.ac.uk
}

of immersive experiences through Virtual Reality (VR) and Augmented Reality (AR) have revealed the possibility of a mixed reality (MR) and screenless future in the context of ubiquitous computing [1-4]. Furthermore, Natural User Interface (NUI) became one of the emerging interactions approaches and interfaces due to the rise of ubiquitous / pervasive technologies $[5,6]$. Nevertheless, the accessibility for these technologies was not introduced sufficiently to people with physical disabilities. Although some approaches [7, 8] were introduced, the constraint of the limited body part-in our case, hands-and the methods of existing gesture recognition [5, 9] needs to be explored further. Thus, we think inclusive design needs a new approach for people with physical disability in relation to assistive technologies. This project introduces a customizable inclusive interface for disabled people who are difficult to interact with spatial computing environment.

\section{INCLUSIVE DESIGN VS CUSTOMIZABLE DESIGN?}

Since the era of 2D interface, accessibility and inclusivity have been considered as one of the key important factors in developing user interactions/interfaces [11]. Researchers working on inclusive HCI have attempted to open various social opportunities to disabled people by improving connectivity and interactions $[12,13]$. For example, one system uses breath as a way of input for interactions [14] while another substitutes vision by tactile images [15]. Although these approaches provide accessible interactions for people with certain disabilities, they lack generality as disability is often highly individual. For instance, many inclusive design projectsincluding the previous examples-are aiming for small groups of people/audience, which require designers to develop different types of systems to embrace people's diverse yet individual conditions [13].

We are entering the era of 3D spatial interaction that will often require people to interact within virtual environments, and people with disability would not be an exception. In this context, we thought designing/proposing a system for people with different individual disabilities is necessary in order to expand the idea of inclusive design. Unlike traditional inclusive design where designers/researchers/engineers need to propose a system that could embrace different types of users, we instead want different users to adapt to one flexible and customizable system. In this paper, we introduce an inclusive NUI system that enables people with physical disability to access 3D interactions via customizable and wearable 


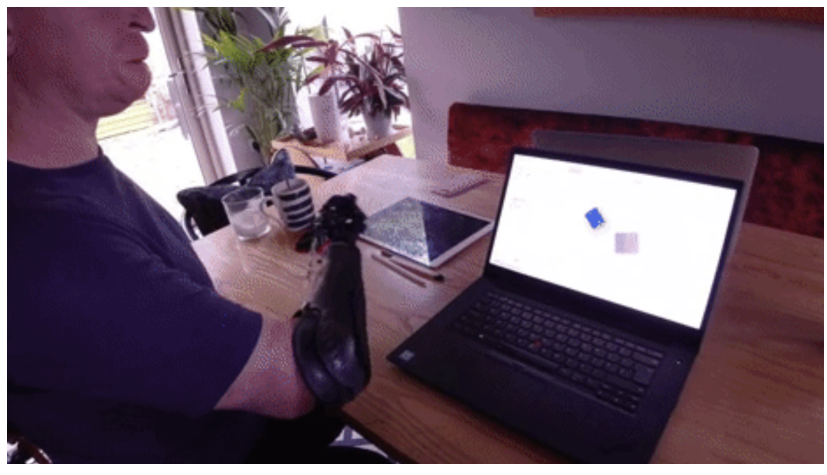

Figure 1: Experiment with a target user

embodied interface. The next subsections provide instructions on how to insert figures, tables, and equations in your document.

\section{STUDIES AND FINDINGS}

To explore the possibility of an inclusive NUI for people who have physical disabilities, several studies were conducted. The goal of these studies was to extract and understand full body movementbased interactions in relation to 3D object manipulations and interactive system/calibration development.

For our studies (figure 1), a total of ten able-bodied subjects (five males and five females, mean age: 24 years, standard deviation of age: 1.1 years) were recruited. In our first study, participants were asked to use their full body to perform four basic interactions-selection, positioning, rotation, and scaling [10]-for 3D object manipulation with their constricted body parts. This initial study/observation was to understand the ways in which people accomplish the four basic tasks in relation to their body movements. In the second study, limitations were added that required participants to use assigned body parts (e.g, right arm and head, or mouth) to conduct the same task as the first experiment. This study was a tentative step to measure and develop an initial calibration system that may be useful for developing prototypes. In addition, we ran the same experiment with two target users-one quadruple amputee, one paralyzed patient with limited motor ability of upper limbs-in order to observe the actual limitations for advanced study.

Throughout this study, we observed and learned that most of the interactions and manipulations are possible via two points in $3 \mathrm{D}$ space. Below is the two-points model we built during this study (figure 2).

Two-Points Model,

- Selection-two points quickly approaching each other.

- Positioning-one point keeps still and the other moves.

- Scaling-two points leave or approach each other at the same time.

- Rotation-two points rotate around the pivot point.

The purpose of building this model was to help us to understand how human body movements in different conditions could express their intentions of interactions using certain body parts in relation to 3D object manipulation. Understanding how people interact was necessary to design an inclusive interface that assists people with physical disabilities.

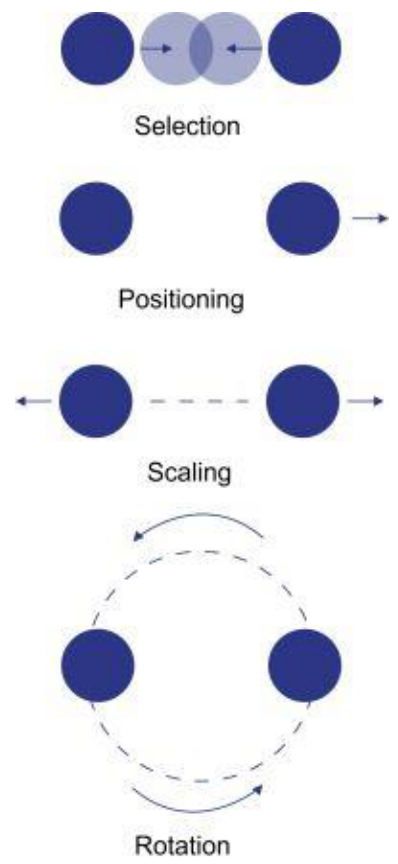

Figure 2: Two-points model. We found that interaction in spatial environment could be described as the relative motion of two points.

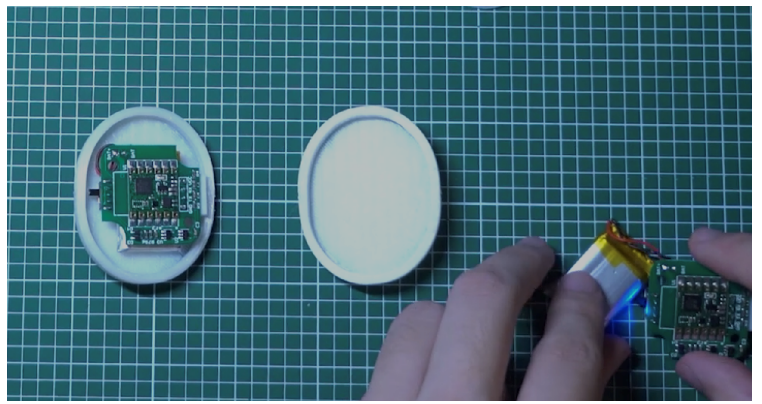

Figure 3: The Dots system contains two body trackers and one charging base.

\section{PROJECT DOT}

Dots is a customizable body interface or system for the future of inclusive pervasive computing. Dots is composed of a wireless charger, sensors and two dot-like slices (figure 3). Users-people with physical disabilities-can have full control with MR interfaces and IoT devices. The two-points model (figure 2) was used to develop this experiential prototype.

To use Dots, users need to first attach two dots onto any of their body parts depending on their body conditions. Users need to make sure the two body parts can make relative movements to accomplish at least one interaction pattern (figure 4). Two dots are also possible to be attached to any object such as table. This depends on the types of interaction users want to achieve. 

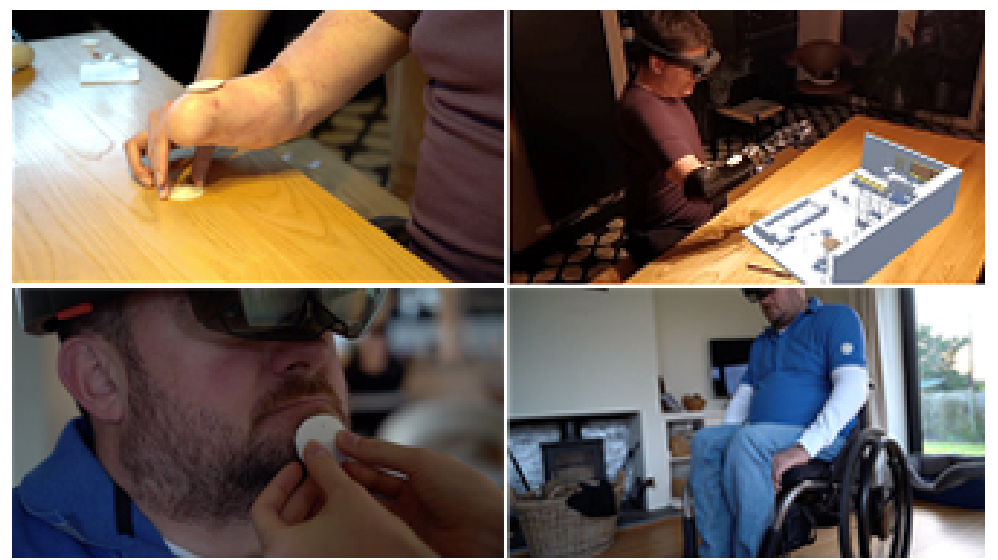

Figure 4: Some use cases of the Dots. This inclusive interface enables people with disability to interact with the Mixed Reality and Internet of Things by leveraging their own body parts and even the environment surrounding them. https://youtu.be/ s8dWghnvQqo

The two dots respectively consist of one IMU sensor, one Bluetooth module and one lithium battery. The dimension of the PCB that contains an IMU sensor and Bluetooth is approximately $15^{*} 15^{*} 2$ $\mathrm{mm}$. The dimension of each dot is $30^{*} 45^{*} 9 \mathrm{~mm}$, which is a sufficient size to contain all components.

These two IMU sensors can measure the relative movement of two dots and then further identify the interaction patterns that users wish to make. Dots also has its own calibration system to improve its accuracy to understand user behaviors. The calibration system consists of two parts. Firstly, after users attach two dots on their body, there will be an initial calibration process to measure the moving range of each dot as well as the maximum and minimum distance between two dots. This process could adjust the product to an appropriate sensitivity. Secondly, the calibration system will become more precise over time by collecting data for each successful or failed interaction.

Dots interacts with MR equipment and IoT devices and allows users to accomplish multiple tasks through body interactions-it consists of one Bluetooth module, which enables it to communicate with any IoT devices that have the same communication protocol. For example, it can create 3D arts in the HoloLens, remotely controlling the smart home devices or surfing on the internet. It empowers disabled people to interact with technologies by allowing them to customize their demands by using their environment and body (figure 4).

We have tested our project with ten participants, and they provided some qualitative feedback. Below is some of the feedback participants shared after prototype trial. They mainly shared their experience of interactions.

- Freedom - they enjoyed the freedom to customize the way of using their body parts.

- Novelty - our design brought them a totally new way of thinking and working.

- Equality - they felt that they are on the same page with everyone now.
The 'customizable' feature of Dots tries to push the boundary of inclusive design. It offers a variety of choices for different situations, which largely breaks the barrier of disabled people's physical limitations. The implicit aim of this project is to explore the feasibility of the body interface within the context of NUI. It attempted to demonstrate a solution to how designers, engineers and HCI researchers might include everyone on the path to the future of ubiquitous computing.

\section{DISCUSSION}

There are three elements that we would like to consider in our future work. Firstly, since the efficiency of certain tasks in our current work has been tested in limited scenarios, we would like to conduct further user studies in different scenarios to establish a quantitative study. Secondly, we would like to consider machinelearning aspects to improve the sensitivity of the calibration system to advance/optimize the accuracy of the system. Thirdly, we would like to compare our system to other systems-such as the usage of vocal interface-to better position and demonstrate the effective elements of our system.

\section{CONCLUSION}

The idea of a customizable body interface was conceived by recognizing the intersection between the spatial computing as well as the physical limitation of hand gesture control. We wanted to make the future accessible to everyone by providing an inclusive device where users can adapt to one flexible/customizable system or interface. The Dots project is a late-breaking result toward the future of inclusive design while suggesting one possible solution within the context of NUI. We hope to share our interest and latebreaking result in MobileHCI 2020 and develop the project further. We wish our prototype and demonstration can provoke more ideas around inclusive design and $\mathrm{HCI}$. 


\section{ACKNOWLEDGMENTS}

We thank all the participants in the experiments and insightful suggestions from tutors from Imperial College London and the Royal College of Art (RCA). Special thanks to Alex Lewis and Neil Palmer for valuable feedback.

\section{REFERENCES}

[1] Weiser, M. 1999. The computer for the 21st century. ACM SIGMOBILE Mobile Computing and Communications Review. 3, 3, 3-11.

[2] Jae Yeol, L. et al. 2006. Convergence of Context-Awareness and Augmented Reality for Ubiquitous Services and Immersive Interactions. Computational Science and Its Applications - ICCSA 2006. 466-474.

[3] Dieter, S. and Gerhard, R. 2007. The World as a User Interface: Augmented Reality for Ubiquitous Computing. Location Based Services and TeleCartography. 369391.

[4] Piekarski, W. 2004. Interactive 3D modeling in outdoor augmented reality worlds. The University of South Australia.

[5] Gillies, M. 2019. Understanding the Role of Interactive Machine Learning in Movement Interaction Design. ACM Transactions on Computer-Human Interaction $26,1,1-34$.

[6] Abowd, G. and Mynatt, E. 2000. Charting past, present, and future research in ubiquitous computing. ACM Transactions on Computer-Human Interaction
(TOCHI) 7, 1, 29-58.

[7] Betke, M. 2010. Intelligent Interfaces to Empower People with Disabilities. Handbook of Ambient Intelligence and Smart Environments. 409-432.

[8] Ann, O. and Theng, L. 2014. A Facial Expression Mediated Natural User Interface Communication Model for Children with Motor Impairments. Assistive Technologies and Computer Access for Motor Disabilities, 254-284.

[9] Trigueiros, P. et al. 2012. "A comparison of machine learning algorithms applied to hand gesture recognition," the Iberian Conference on Information Systems and Technologies (CISTI 2012), 1-6.

[10] LaViola, J. 2017. 3D user interfaces. Addison-Wesley Professional.

[11] Persson, H. et al. 2014. Universal design, inclusive design, accessible design, design for all: different concepts-one goal? On the concept of accessibilityhistorical, methodological and philosophical aspects. Universal Access in the Information Society. 14, 4, 505-526.

[12] Fitch, D. 2002. Digital inclusion, social exclusion and retailing: an analysis of data from the 1999 Scottish Household Survey. IEEE 2002 International Symposium on Technology and Society (ISTAS'02). Social Implications of Information and Communication Technology. Proceedings (Cat. No.02CH37293). 309 (2002), 313.

[13] Abascal, J. and Nicolle, C. 2005. Moving towards inclusive design guidelines for socially and ethically aware HCI. Interacting with Computers. 17, 5, 484-505

[14] Aziz, A. et al. 2008. The flote. Proceedings of the 10th international ACM SIGACCESS conference Computers and accessibility - Assets ' 08 .

[15] Rita, B, Y, P, Collins, C.C, Saunders, F.A, White. B and Scadden, L. 1969. Vision Substitution by Tactile Image Projection. Nature. 221(5184), 963-964. 